\title{
GEANT4 APPLICATIONS IN SPACE
}

\author{
MAKOTO ASAI \\ Stanford Linear Accelerator Center, Stanford University \\ Menlo Park, California 94025, U.S.A.
}

\begin{abstract}
Use of Geant4 is rapidly expanding in space application domain. I try to overview three major application areas of Geant4 in space, which are apparatus simulation for pre-launch design and post-launch analysis, planetary scale simulation for radiation spectra and surface and sub-surface explorations, and micro-dosimetry simulation for single event study and radiation-hardening of semiconductor devices. Recently, not only the missiondependent applications but also various multi-purpose or common tools built on top of Geant4 are also widely available. I overview some of such tools as well. The Geant4 Collaboration identifies that the space applications are now one of the major driving forces of the further developments and refinements of Geant 4 toolkit. Highlights of such developments are introduced.
\end{abstract}

\section{Apparatus simulation}

Apparatus simulation is essential for both pre-launch design studies and postlaunch analyses. Geant4 [1,2] is widely and commonly used for various current and future science missions including XMM-Newton [3], GLAST [4], LISA and LISA Pathfinder [5], RHESSI [6], ACE [7], JWST [8], INTEGRAL [9], Bepi Colombo [10], Messenger [11], Herschel [12], Cassini and Huygens [13], AstroE2 [14], ConeXpress [15], SELENE [16], SWIFT [17] and modules of ISS (International Space Station) including Columbus [18], AMS [19] and MAXI [20]. In the following sections, I will overview how Geant4 is used for some of these missions.

\subsection{GLAST LAT}

GLAST[4] is to be launched in early 2008 and measure the direction, energy and arrival time of celestial gamma rays. The LAT (Large Area Telescope) instrument measures gamma rays in the energy range from about $20 \mathrm{MeV}$ to greater than $300 \mathrm{GeV}$. In addition, the GBM (Gamma-ray Burst Monitor) instrument provides correlative observations of transient events in the energy range between $20 \mathrm{KeV}$ and $20 \mathrm{MeV}$. GEANT4 was adopted since 1999 for the test beam simulations and the balloon flights [21].

Invited talk presented at 10th ICATPP Conference On Astroparticle, Particle, Space Physics, Detectors And Medical Physics Applications, 8-12 Oct 2007, Como, Italy 


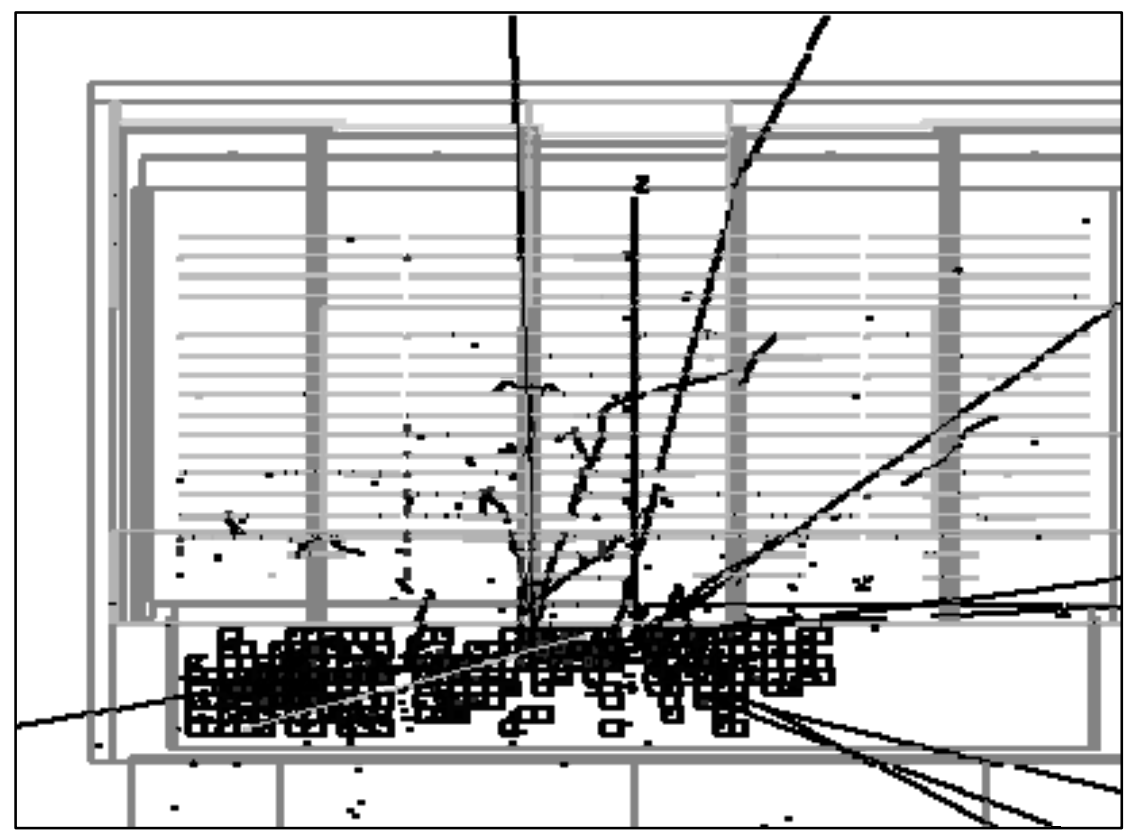

Figure 1. Simulated background hadronic event in GLAST LAT[4]. Courtesy of F. DeBois (SLAC) and F. Longo (INFN/Trieste).

For GLAST mission, simulation of back ground events is even more important than simulation of signal events. Figure 1 shows a proton incident hadronics event in CsI crystal calorimeter which causes EM particles leak into silicon tracker and fakes as a signal event. They are now processing for 50 billion background events of cosmic proton, neutron and electron and earth albedo backgrounds prior to proceed to simulating one full year signal events.

\subsection{Cassini LEMMS}

LEMMS (Low Energy Magnetospheric Measurement System) is on board of Cassini spacecraft to measure the energy and spatial distribution of energetic particles (electrons and ions separately) in the interplanetary medium and in the magnetosphere of Saturn. LEMMS consists of low energy detector head with collimator, high energy detector head with collimator, and programmable turntable. The measurements of energetic particles are based on the loss of energy in semiconductor detectors. LEMMS has 11 different detectors. These detectors and collimators are modeled in Geant 4 and studied as shown in Fig.2 [14]. 

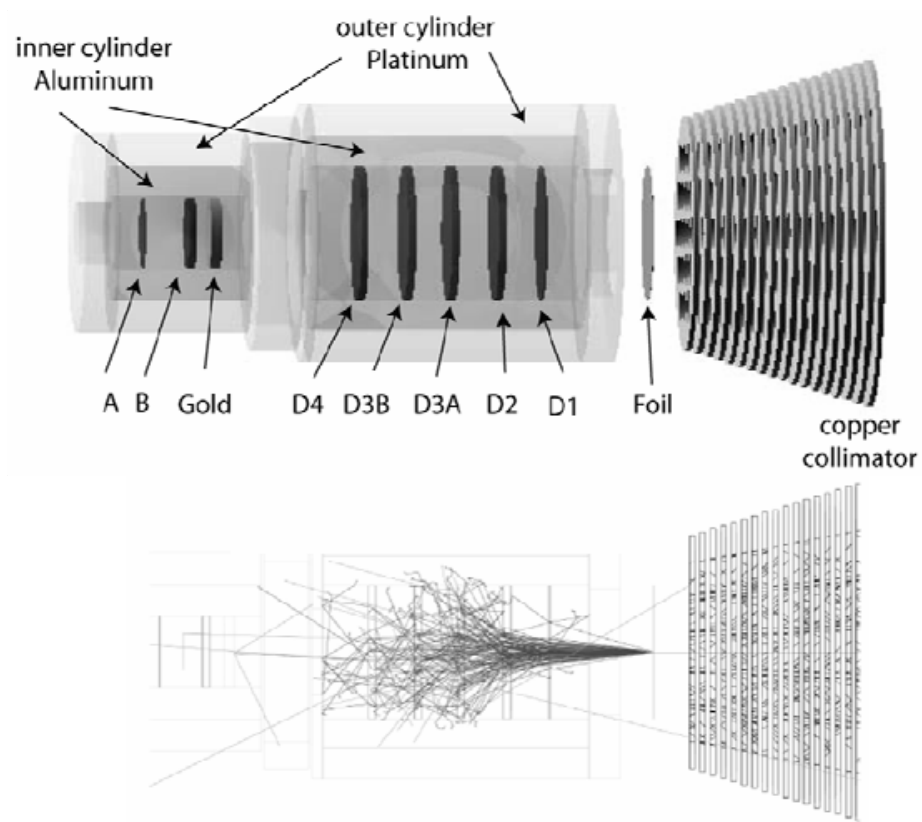

Figure 2. Geant4 geometry model of LEMMS and simulation of $2 \mathrm{MeV}$ electron pencil beam. Courtesy of D.K. Haggerty (JHUAPL).

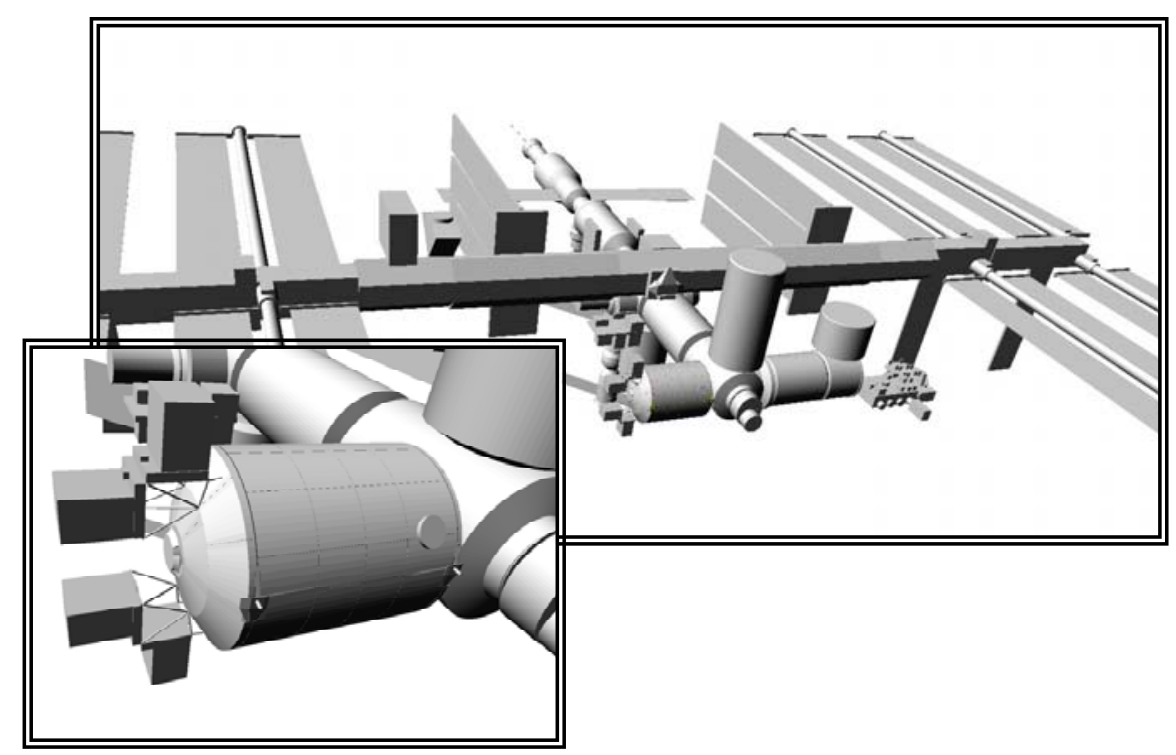

Figure 3. Geant4 geometry model of International Space Station (up) and the Columbus module (left). Courtesy of T. Ersmark (KTH Stockholm). 


\subsection{ISS Columbus}

Full geometry model of ISS (International Space Station) and particularly detailed apparatus structure of European Columbus module are built to accurately calculate radiation fluxes and doses to astronauts inside the Columbus module [18]. Incident radiation includes trapped protons, galactic cosmic rays, solar particle events and Earth albedo neutrons.

\section{Planetary scale simulation}

Planetary scale simulation is essential for both radiation spectra calculation and surface and sub-surface explorations. Full-scale geometrical and magnetosperic structure of a planet is modeled in Geant 4 to simulate the trapped solar particles to the Earth [22], Mercury [23], Mars [24] and Jupiter [25]. Geant4 is also used successfully for simulation of even larger scale and in higher energies such as particle accelerations in solar flare [26] and gamma-ray burst [27].

Interactions of solar particles on the (sub-)surface are simulated with Geant4 for mineralogical survey of Mercury for Bepi Colombo mission [28] and for water search of Moon for SELENA mission [29].

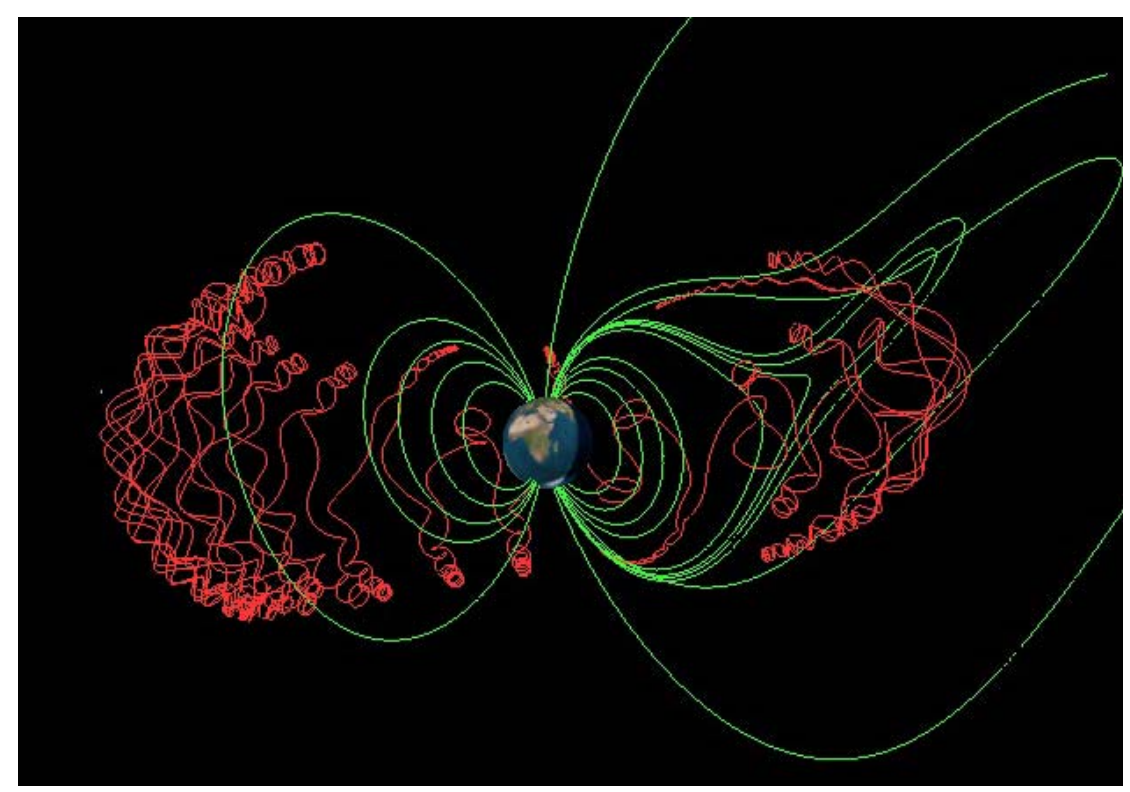

Figure 4. Simulated trajectory of a proton trapped in the Earth magnetosphere. Courtesy of L. Desorgher (U. Bern). 


\section{Micro-dosimetry simulation}

Three sources in natural space environment contribute to various effects on microelectronics. These are solar particles (protons and heavier ions), galactic cosmic rays, and particles trapped in the Earth's radiation belts. Due to these radiations, there are three basic effects that occur when components are exposed, single event effects (SEEs) [30], total ionization dose and charge deposit [31], and displacement damage [32].

Single event upset (SEU) is one of SEEs. SEU observations recorded by ISS show clear patterns of temporal and special dependence. SEUs are mainly due to two ionization cases, direct ionization caused by incident particle and indirect ionization caused by secondary particles generated by the interaction of the primary particle. In all cases, charge collected on a sensitive node of electrical circuit causes unwanted change in the information stored in the circuit. Commercial Technology Computer Aided Design (TCAD) [33] tool alone is unable to predict indirect ionization case. Also, classical method with CREME96 [34] does not give satisfactory results [35]. Combining Geant4based Monte-Carlo Radiative Energy Deposition (MRED) tool with TCAD and SPICE, energy partitioning information simulated by MRED is transferred to TCAD to model the electron-hole transportation, and then whole-circuit response of SEU is calculated by SPICE [36]. Similar approach is taken by other applications [37].
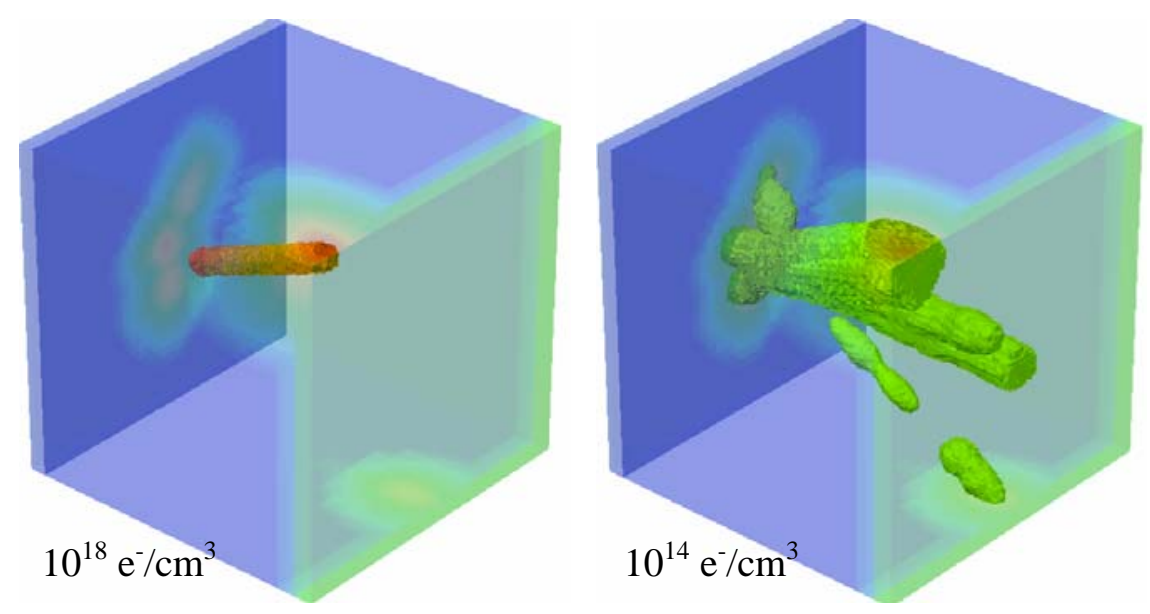

Figure 4. Electron density distribution caused by secondary particles of proton-tungsten interaction simulated by MRED. Courtesy of R. Reed (Vanderbilt Univ.) . 


\section{General-purpose tools}

There are many general / multipurpose tools built on top of Geant 4 available for space applications. These include the following tools.

- General Framework

- GRAS [38]

- Plug-in Physics Model

- G4LECS [39]

- Sector Shielding Analysis

- MULASSIS [40]

- Open Frontier [41]

- Radiation on planets

- PLANETOCOSMICS [42]

- Dosimetry on semiconductor devices

- MRED [36]

- GEMAT [43]

- FASTRAD [44]

Geant4 offers flexibility and robustness of kernel, powerful capability of geometrical modeling and comprehensive coverage of physics models, which are all essential to these diverse variations of application tools.

\section{Recent developments in Geant 4 toolkit}

The Geant4 Collaboration [45] identifies that the space application domain is now one of the major driving force of the further developments and refinements of Geant4 toolkit. In this section I introduce some of such developments which are driven mainly, if not solely, by the requirements from the space application domain.

\section{1. $\quad$ Tessellated solid}

Tessellated solid is a kind of solid newly supported by Geant4 since 2006. It is a solid consists of arbitrary number of facets. Each facet can be triangular or quadrangular. This solid shape is of particular importance for conversion from CAD system bounded with generic surfaces into an approximate description with facets of defined dimension (Fig. 5).

To import geometrical models from CAD, it is required to convert first the CAD shapes into tessellated surfaces. A way to do this is to save the shapes in the geometrical model as STEP [46] files and convert them using a tool like STViewer [47] to tessellated (faceted surfaces) solids. This strategy allows to 
import any shape with some degree of approximation; the converted CAD

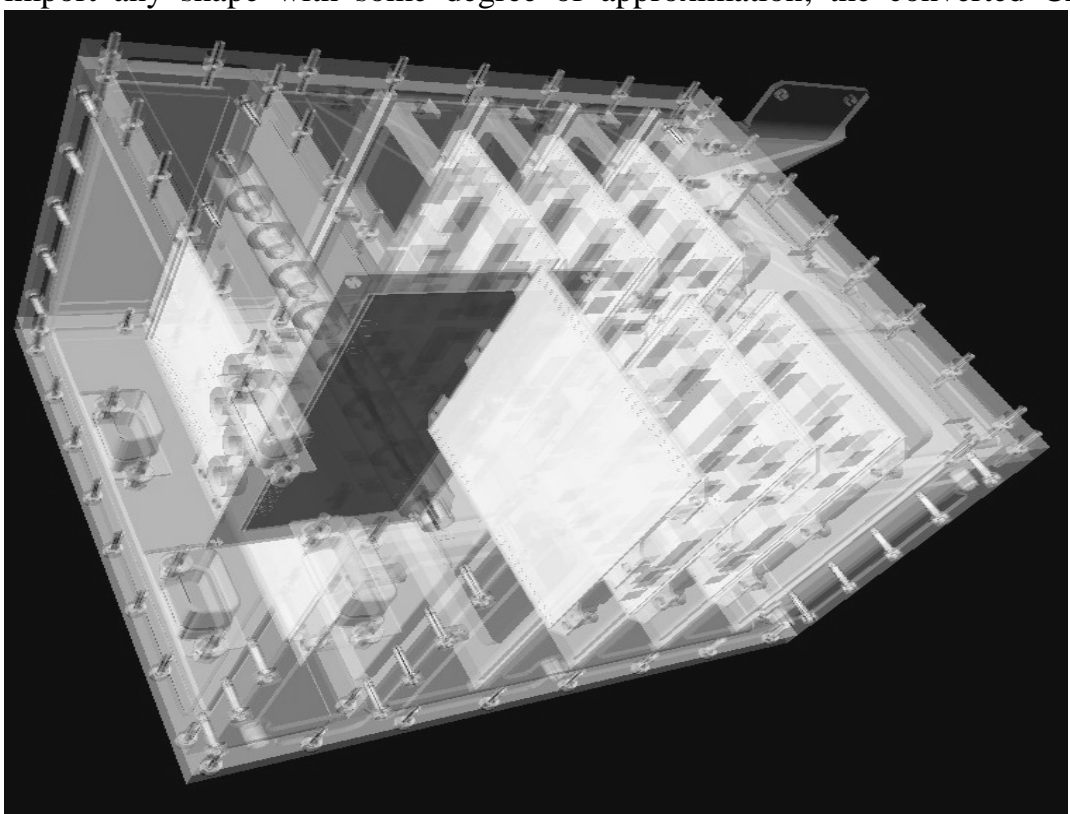

Figure 5. A module geometry imported from CAD into Geant4 tessellated solid. Courtesy of G. Cosmo (CERN)

models can then be imported through GDML (Geometry Description Markup Language) [48] into Geant4 and be represented as tessellated solid shapes.

\subsection{Quantum Molecular Dynamics code}

Radiation in space includes considerable heavy nuclides and they are in particular important for simulating dose and also SEEs. Geant4 currently has Binary Light Ion Cascade model [49] and Wilson Abrasion and Ablation model [50], both of which work mainly for relatively light nucleus [51]. By the end of 2007, Geant4 is releasing its new Quantum Molecular Dynamics (QMD) model [52], which firstly works for most nuclides of up to $300 \mathrm{MeV} / \mathrm{n}$ (Fig. 6), and it is foreseen to be extended to several $\mathrm{GeV} / \mathrm{n}$.

\subsection{Built-in scoring tools}

For recording detector responses, Geant 4 originally offered only the abstract classes with various ready-to-reuse examples. Thus, the user had to implement his/her detector responses. It is a reasonable requirement for a large-scale HEP 


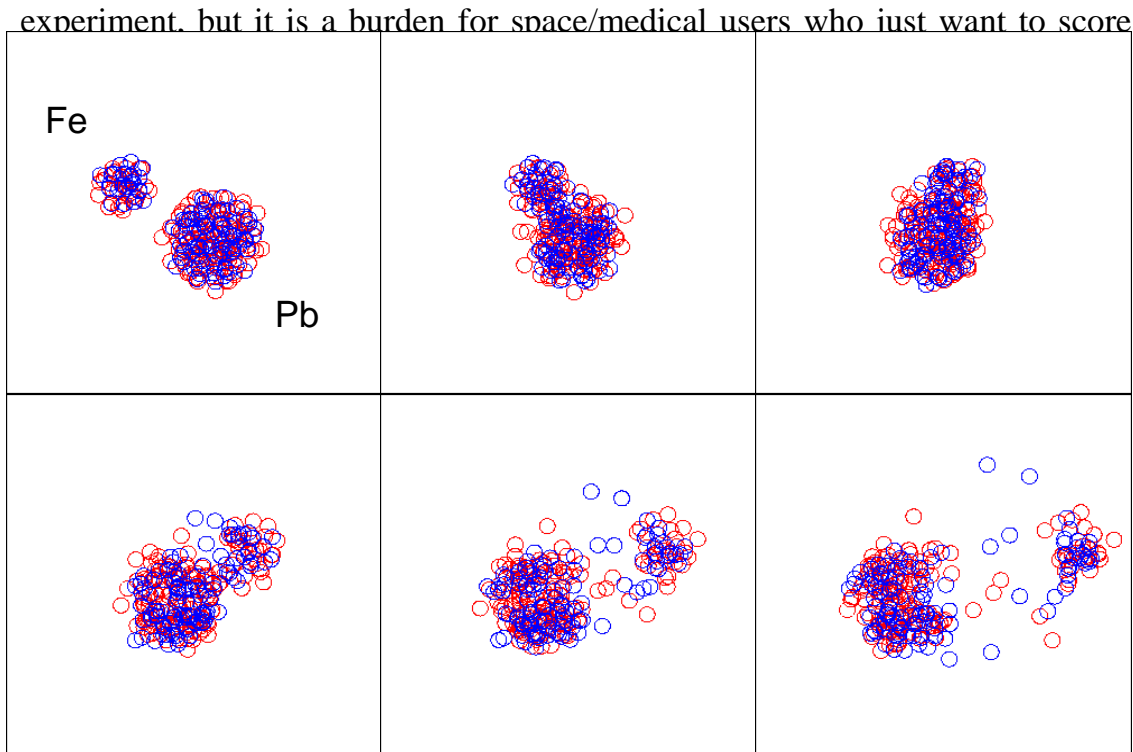

Figure 5. A collision of $290 \mathrm{MeV} / \mathrm{n}$ iron onto lead simulated by Geant4 QMD code. Blue circle represents proton and red circle represents neutron. Courtesy of T. Koi (SLAC)

most common quantities such as dose or flux. By the end of 2005, Geant4 released built-in concrete implementation of scorers for common quantities, which include energy, dose and charge deposition, surface flux and current, and number of secondary generation [53]. It is foreseen that all of such scorers will be able to define to arbitrary scoring mesh with simple interactive commands regardless of the geometrical model of actual materials.

\section{Conclusions}

In 2008, Geant 4 will be in 10 years of its public releases. It had been addressing mainly to the requirements from HEP experiments, and Geant4 is nowadays well adapted to most of the current and future HEP experiments as their simulation engine [54]. Use of Geant4 is quite rapidly expanding in space application domain. I gave an overview of such Geant4 applications, in particular for apparatus simulation, planetary scale simulation, micro-dosimetry simulation and general-purpose tools for space missions. I have to note here that my overview is quite incomplete, and much richer list of Geant4 applications in space domain can be found at the Geant 4 Space Users home page [55]. Robust and flexible kernel structure, comprehensive coverage of physics models and 
powerful geometrical modeling capability provided by Geant 4 were proven to be suitable for mission critical simulations.

\section{References}

1. S. Agostinelli et al., NIM A506, 250 (2003)

2. J. Allison et al., IEEE Trans. Nucl. Sci 53, 270 (2006)

3. R. Nartallo et al., IEEE Trans. Nucl. Sci 48, 1815 (2001)

4. L. Baldini et al., Nucl. Phys. Proc. Suppl. 150, 62 (2006).

N. Omodei et al., AIP Conf. Proc. 906, 1 (2007)

R.M. Kippen et al., AIP Conf. Proc. 921, 590 (2007)

5. M. Henrique et al., Astropart. Phys. 22, 451 (2005)

H.M. Araujo, et al., Class. Quantum Grav. 20, S311 (2003)

6. C. Wigger et al., Astrophys. J. 613, 1088 (2004)

7. D.K. Haggerty et al., Advances in Space Research 32, 423 (2003)

8. G. Santin et al., IEEE Trans. Nucl. Sci 52, 2294 (2005)

9. C. Ferguson et al., presentation at $4^{\text {th }}$ INTEGRAL workshop (2000).

10. A. Owens et al., presentation at Round Table on 21st Century Monte Carlo Methods for Space Applications, Noordwijk, 2001

11. D.K. Haggerty, presentation at Conference of Solar and Space Physics and the vision for Space Exploration (2005)

12. C. Bongardo, et al., Exp. Astro. 21, 67 (2006)

13. D.K. Haggerty et al., Advances in Space Research 33, 2303 (2004)

14. M. Ozaki et al., IEEE Trans. Nucl. Sci 53, 1310 (2006)

15. http://space-env.esa.int/ProjectSupport/ConeXpress/ConeXpress.htm

16. O. Okudaira et al., Proceedings of $9^{\text {th }}$ Conference on Astroparticle, Particle and Space Physics, Detectors and Medical Physics Applications, 490 (2005)

17. M. Suzuki et al., IEEE Nucl. Sci. Symp. Conf. Rec. vol.5, 3550 (2003)

18. T. Ersmark et al., IEEE Trans. Nucl. Sci 51, 1378 (2004)

T. Ersmark, Ph.D. thesis, ISBN 91-7178-398-9, KTH Stockholm, June 2006

19. M.C. Espirito-Santo et al., IEEE Trans. Nucl. Sci. 51, 1373 (2004)

20. H. Tomida et al., Proc. SPIE vol.4851, 993 (2003)

21. T.H. Burnett et al., IEEE Trans. Nucl. Sci .49, 1898 (2002)

22. L. Desorgher et al., Proceedings of $28^{\text {th }}$ International Cosmic Ray Conference, 4281 (2003)

F. Lei et al., IEEE Trans. Nucl. Sci 51, 3442 (2004)

M.D. Looper et al., Presentation at $4^{\text {th }}$ Geant4 Space Users' Workshop, (2005)

23. M. Gurtner et al., Advances in Space Research 37, 1759 (2006)

24. A. Keating et al., IEEE Trans. Nucl. Sci 52, 2287 (2005)

25. R.F. Elsner et al., Icarus 178, 417 (2005) 
26. J. Kotoku et al, e-Print: arXiv:0708.0057 (2007)

27. K. Murase and S. Nagataki, Phys. Rev. D73:063002 (2006)

28. A. Mantero et al., IEEE Nucl. Sci. Symp. Conf. Rec. vol.3, 1527 (2003)

29. K. Hayatsu et al., Presentation at $10^{\text {th }}$ Conference on Astroparticle, Particle and Space Physics, Detectors and Medical Physics Applications (2007) N. Yamashita et al., Presentation at $10^{\text {th }}$ Conference on Astroparticle, Particle and Space Physics, Detectors and Medical Physics Applications (2007)

30. P.E. Dodd et al., IEEE Trans. Nucl. Sci 50, 583 (2003)

31. T.R. Oldham and F.B. McLean et al., IEEE Trans. Nucl. Sci 50, 483 (2003)

32. J.R. Srouor et al., IEEE Trans. Nucl. Sci 50, 653 (2003)

33. http://www.synopsys.com/products/tcad/tcad.html

34. A.J. Tylka et al., IEEE Trans. Nucl. Sci 43, 2758 (1996)

35. R.A. Reed et al., IEEE Trans. Nucl. Sci 50, 622 (2003)

36. K.M. Warren et al., IEEE Trans. Nucl. Sci 53, 2125 (2005)

A.S. Kobayashi et al., IEEE Trans. Nucl. Sci 53, 2189 (2003)

37. I. Jun et al, JPL R\&TD Annual Report 06-128, Pasadena, CA (2006)

38. G. Santin et. al., IEEE Trans. Nucl. Sci 52, 2294 (2005)

39. R.M. Kippen, New Astronomy Reviews 48, 221 (2004)

40. F. Lei et al., IEEE Trans. Nucl. Sci 49, 2788 (2002)

41. H. Sdunnus et al., Presentation at $20^{\text {th }}$ European Workshop on Thermal and ECLS Software (2006)

42. http://cosray.unibe.ch/ laurent/planetocosmics/

43. G. Santin et al., Nuclear Physics B - Proceedings Supplements vol.125, 69 (2003)

44. T. Beutier et al., Proceedings of $7^{\text {th }}$ European Conference on Radiation and its Effects on Components and System, 181 (2004)

45. http://cern.ch/geant4/

46. http://www.tc184-sc4.org/SC4_Open/SC4_Standards_Developers_Info/ Files/ STEP_application_handbook_63006.pdf

47. http://www.steptools.com/products/stviewer/

48. R. Chytracek et al., IEEE Trans. Nucl. Sci 53, 2892 (2006)

49. G. Folger et al., Eur. Phys. J. A21, 407 (2004)

50. P. Truscott et al., Proceedings of Monte Carlo 2005 Topical Meeting ISBN:0-89448-695-0 (2005)

51. T. Koi et al., Proceedings of Monte Carlo 2005 Topical Meeting ISBN: 0-89448-695-0 (2005)

52. J.Z.H. Zhang, ISBN 981-02-3388-4, (1998)

53. T. Aso et al., IEEE Nucl. Sci. Symp. Conf. Rec. vol.2, 978 (2005)

54. http://geant4.web.cern.ch/geant4/collaboration/Geant4-Review2007.html

55. http://geant4.esa.int/ 